\title{
PORE-SCALE MODELING OF TRANSIENT AND STEADY-STATE VAPOR DIFFUSION IN PARTIALLY-SATURATED POROUS MEDIA
}

\author{
Stephen W. Webb \\ Mission Analysis \& Simulation Department 6524 \\ Sandia National Laboratories \\ Albuquerque, New Mexico 87185-0977, USA
}

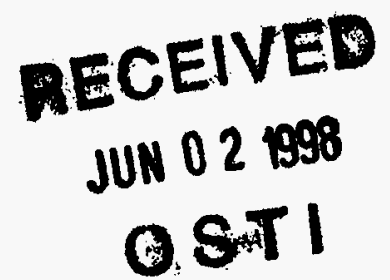

\begin{abstract}
Vapor diffusion in porous media in the presence of its own liquid may be enhanced due to pore-scale processes, such as condensation and evaporation across isolated liquid islands. Webb and Ho (1997) developed a mechanistic pore-scale model of these processes under steady-state conditions in which condensation and evaporation on the liquid island were equal. The vapor diffusion rate was significantly enhanced by these liquid island processes by up to an order of magnitude compared to a dry porous media. However, vapor transport by diffusion is often complicated by transient effects, such as in drying applications, in which net evaporation of liquid may further augment the vapor flux from diffusion. The influence of transient effects on the enhancement factors for vapor diffusion is evaluated in this paper. In addition, the effect of vapor pressure lowering on the enhancement factor and on porescale vapor fluxes is shown.
\end{abstract}

\section{NOMENCLATURE}

C specific heat

$\mathrm{D}^{\mathrm{K}} \quad$ Knudsen diffusion coefficient

$\mathrm{D}_{12} \quad$ binary diffusion coefficient for water vapor and air

$\mathrm{f}_{\mathrm{VPL}} \quad$ vapor pressure lowering factor

F mass flux

g $\quad$ gravity

h enthalpy; coordinate measured from the center of the channel

J mole flux

k permeability

K thermal conductivity

M mass accumulation term; molecular weight

n normal vector

P pressure

q source

$\mathrm{r}$ pore radius

$\mathrm{R} \quad$ universal gas constant; particle radius

S saturation

t time

$\mathrm{T}$ temperature

u internal energy

$\begin{array}{ll}\text { V } & \text { volume } \\ \mathbf{X} & \text { mole fraction; linear dimension } \\ \text { Greek } & \\ \beta & \text { porous media factor; phase indicator } \\ \rho & \text { density } \\ \kappa & \text { component } \\ \sigma & \text { surface tension } \\ \tau & \text { tortuosity coefficient } \\ \phi & \text { porosity } \\ \omega & \text { mass fraction } \\ \Gamma & \text { surface } \\ \mu & \text { viscosity } \\ & \\ \text { Subscripts } \\ \text { C } & \text { capillary } \\ \text { g } & \text { gas } \\ \text { I } & \text { gas component I } \\ \text { l } & \text { liquid } \\ \text { R } & \text { rock } \\ \text { sat } & \text { saturated } \\ \text { vap } & \text { vapor } \\ \text { W } & \text { water } \\ \kappa, \kappa^{*} & \text { component } \\ & \end{array}$

\section{INTRODUCTION}

Vapor diffusion in porous media may be significant in the flow of subsurface fluids and in the transport of contaminants. In many environmental remediation and waste isolation applications, such as removal of nonaqueous phase liquid (NAPL) contaminants from lowpermeability layers in the subsurface, vapor diffusion is the limiting transport mechanism (Ho and Udell, 1992, Webb and Phelan, 1997). Diffusion of vapors may also be important in high-level nuclear waste repositories, in the drying of paper and textiles, and in evaporation from soils.

Gas diffusion in porous media is generally significantly smaller than 


\section{DISCLAIMER}

This report was prepared as an account of work sponsored by an agency of the United States Government. Neither the United States Government nor any agency thereof, nor any of their employees, makes any warranty, express or implied, or assumes any legal liability or responsibility for the accuracy, completeness, or usefulness of any information, apparatus, product, or process disclosed, or represents that its use would not infringe privately owned rights. Reference herein to any specific commercial product, process, or service by trade name, trademark, manufacturer, or otherwise does not necessarily constitute or imply its endorsement, recommendation, or favoring by the United States Government or any agency thereof. The views and opinions of authors expressed herein do not necessarily state or reflect those of the United States Government or any agency thereof. 
in free space due to the presence of the porous medium. The flow area for gas-phase diffusion is reduced by the presence of the solid particles, by the presence of any liquid, and by the fact that the flow path for diffusion in a porous medium is more tortuous than in free space. Using Fick's law, gas diffusion in a porous media may be expressed as follows

$$
F_{i}=-\tau \phi S_{g} D_{12} \rho_{g} \nabla \omega_{i}=-\beta D_{12} \rho_{g} \nabla \omega_{i}
$$

where $D_{12}$ is the free-space diffusion coefficient at the pressure and temperature of interest. The product of the tortuosity coefficient, $\tau$, the porosity, $\phi$, and the gas saturation, $S_{\mathrm{g}}$, is often referred to as the porous media factor, $\beta$. Because the tortuosity coefficient and the porosity are always less than 1 , and the gas saturation is a maximum of 1 , the porous media factor, $\beta$, can be much less than 1 , and gas diffusion in a porous medium is usually significantly lower than in free space. Vapor diffusion is often analyzed similar to gas diffusion such that vapor diffusion in porous media is also significantly less than in free space. However, it has also been postulated that diffusion of a condensible vapor in the presence of its liquid may be considerably enhanced compared to gas diffusion rates and may approach or even exceed free-space values. (In the present discussion, gas refers to a non-condensible inert gas under the conditions of interest, or air. Vapor refers to the gas phase which may have a liquid phase present, or water vapor).

Enhanced vapor diffusion was first considered by Philip and deVries (1957) for soils. Jury and Letey (1979) estimated that the value of $\beta$ is of the order 1.0 resulting in considerable enhancement of vapor diffusion compared to gas diffusion. When $\beta$ equals 1.0 , diffusion is not affected by the porous medium at all and is equal to the value for free-space diffusion. Cary (1979) also developed a model for the enhancement factor with a similar magnitude to that of Jury and Letey (1979).

Cass et al. (1984) inferred enhancement factors from thermal conductivity data for sand and silt loam and developed theoretical predictions of the general behavior of theoretical enhancement factors. Their data agree reasonably well with the models of Jury and Letey (1979) and Cary (1979) at intermediate liquid saturations. However, there are other regions of significant differences between these models and the data, especially at low liquid saturations.

The mechanisms for such an enhancement are postulated to include local condensation and evaporation at isolated liquid "islands" within the porous medium. Instead of forming a barrier to vapor diffusion, these liquid islands enhance the vapor diffusion rate, or apparent diffusion rate, by reducing the effective diffusion path length. Due to local vapor pressure lowering, condensation occurs at the upstream end of the island, while evaporation occurs on the downstream end, even for isothermal conditions. The difference in curvature of the meniscus at each end of the liquid island, and in the capillary pressure, will result in a liquid pressure difference across the liquid island causing liquid flow.

Another mechanism postulated to contribute to this enhancement is an increased temperature gradient in the gas phase compared to the average temperature gradient in the equivalent porous medium due to the lower conductivity of the gas phase. This increased temperature gradient mechanism will not be discussed in the present study because no temperature gradient is imposed. This contribution will be addressed in subsequent work.

Ho and Webb (1998) have reviewed enhanced vapor diffusion mechanisms, models and data. They noted that enhanced vapor diffusion has been inferred from other data, such as thermal conductivity or total water (liquid plus vapor) flow; no direct measurements of enhanced vapor diffusion have been performed. They used a simple pore-scale model to show that enhanced vapor diffusion is indeed possible through condensation/evaporation processes across liquid islands. Based on this review, additional experiments and modeling at multiple length scales were proposed to try to understand the mechanisms involved.

As a result of this review, experiments are currently underway including tests with individual pores, a two-dimensional pore network, and a three-dimensional porous medium. Data for the porous medium are reported by $\mathrm{Gu}$ et al. (1998) and indicate significant enhancement of vapor diffusion. The other experiments are in progress and data are not available at the present time.

Additionally, Webb and Ho (1997) performed pore-scale modeling of steady-state vapor diffusion in porous media including the interactions of the vapor with the liquid islands and vapor pressure lowering. The Dusty-Gas Model was used to simulate air-vapor advection and diffusion in a pore network including Knudsen and ordinary (Fickian) diffusion; surface diffusion effects were not considered. Kelvin's equation was used to estimate vapor pressure lowering effects at the liquid island gas-liquid interface, and the Young-Laplace equation was used to evaluate gasliquid pressure differences at either end of the liquid island. Concentration gradients were applied to the pore-scale model to calculate the vapor and air flow rates which were then compared to pure gas diffusion in the porous medium and in free space. No temperature or pressure gradients were imposed. Three different models were considered in their numerical assessment of enhanced vapor diffusion mechanisms. The results of their modeling indicated that significant enhancement of vapor diffusion is possible at the pore scale due to the condensation/evaporation mechanisms under steady-state conditions. The degree of enhancement (compared to all-gas conditions) is up to an order of magnitude in their simulations.

Vapor diffusion in porous media can occur under steady-state or transient conditions. In steady-state, as considered by Webb and $\mathrm{Ho}$ (1997), the vapor mass flux into and out of the model are equal. The model boundary conditions are centered around the equilibrium vapor concentration of the liquid island, which is at a lower vapor pressure than saturation due to vapor pressure lowering effects. Flow in the liquid island is at steady state such that the condensation and evaporation rates at the ends of the liquid island are the same and are equal to the liquid flow rate through the liquid island due to capillary pressure differences.

Vapor diffusion can also exist under transient conditions, as exemplified by drying applications. In drying, the boundary conditions may not be centered around the liquid island equilibrium conditions, and significant net evaporation of vapor from the liquid island may occur. Net evaporation or condensation would change the vapor mass flux across the model such that the inlet and outlet mass flow rates would be different. These conditions present some difficulties in defining an enhancement factor because the vapor flux is spatially variable, which is addressed later in this paper.

In related work, Plumb and Prat (1992) developed a simple porescale numerical model to evaluate the effective vapor diffusion coefficient for drying conditions. They investigated a number of two-dimensional pore-scale models $(5 \times 5,8 \times 8,12 \times 12)$ with variable pore sizes. The initial conditions of the model were due to drainage, so no global liquid flow occurred in their calculations. Vapor pressure lowering effects were ignored, and vapor diffusion was calculated by Fick's law. Drying-type boundary conditions were imposed such that transient vapor diffusion occurred. Vapor diffusion varied significantly with the model size and the liquid saturation. The calculated diffusivity was enhanced up to a 
factor of 30 compared to dry conditions. This enhancement was based on the outlet model vapor flow rate (Plumb, personal communication, 1997 ) and is due to the significant evaporation from the liquid islands. This model is discussed in more detail in Prat (1993).

Nowicki et al. (1992) performed calculations similar to Plumb and Prat (1992). They developed a two-dimensional pore-scale model, which was subjected to transient drying boundary conditions. They started out fully saturated with liquid, and global liquid flow occurred. Vapor pressure lowering was considered. Evaporation from the liquid menisci is calculated from an expression given by Bird, Stewart, and Lightfoot (1960) for diffusion through a stagnant gas column. In contrast to the results of Plumb and Prat (1992), their calculations indicate that vapor diffusion is not enhanced relative to the value for the all-gas case; in fact, it is significantly reduced.

The significant difference in the results between Nowicki et al. (1992) and Plumb and Prat (1992) can be explained. In Nowicki et al. (1992), evaporation from the liquid was suppressed compared to Plumb and Prat (1992). For example, the partial pressure of the vapor is less than $15 \%$ of the saturated vapor pressure at small liquid saturations. Therefore, significant vapor pressure lowering of up to $85 \%$ occurred. Unfortunately, the parameters or the fluids used in the Nowicki et al. (1992) calculations were not reported. As calculated by Webb and Ho (1997), vapor pressure lowering would only reduce the vapor pressure of water by a maximum of $0.022 \%$ at $20^{\circ} \mathrm{C}(0.5 \mathrm{~Pa}$ reduction out of a vapor pressure of $2337 \mathrm{~Pa}$ ) for a $5 \mu \mathrm{m}$ pore radius. For vapor pressure lowering of $85 \%$, either the pore radius had to be extremely small, or the difference is due to the evaporation model used by Nowicki et al. (1992). Webb and Ho (1997) did not see significant resistance to vapor diffusion in their calculations as indicated by their vapor pressure profiles, although their calculations were only for small concentration gradients.

In the present study, a number of issues are addressed. First, the effect of the boundary conditions on enhanced vapor diffusion is studied. Webb and Ho (1997) investigated steady-state vapor diffusion, while Plumb and Prat (1992) and Nowicki et al. (1992) studied transient vapor diffusion. An obvious question concerns the differences between vapor diffusion under these two different boundary conditions. The question is also directly applicable to the experiments performed by $\mathrm{Gu}$ et al. (1998), which were not at steady state. Second, the effect of vapor pressure lowering is addressed. In many models such as Plumb and Prat (1992), vapor pressure lowering is ignored due to its assumed small effect. Whitaker (1977), in his derivation of conservation equations for drying, also assumed that vapor pressure lowering could be neglected due to its small magnitude. (In a recent revision of his article, Whitaker has included vapor pressure lowering based on the results of Webb and Ho (1997) (Whitaker, personal communication, 1997)). Steady-state vapor diffusion is driven by vapor pressure lowering as discussed by Webb and Ho (1997). The contribution of vapor pressure lowering to vapor diffusion in the steady-state and transient regimes is therefore discussed.

\section{MODEL DESCRIPTION}

As mentioned above, Webb and Ho (1997) developed a mechanistic pore-scale network model to investigate steady-state enhanced vapor diffusion in porous medium. This model will be used in the present study to investigate the question of transient vs. steady-state enhanced vapor diffusion and to study the influence of vapor pressure lowering. The model is summarized below; additional details are given by Webb and Ho (1997).
Conceptual Model. The conceptual model is depicted in Figure 1. The porous media is considered to be a series of randomly-arranged spheres. Heat transfer occurs between the spheres due to particle-to-particle contact, while flow of gas occurs around the spheres and around any liquid islands present. The liquid saturation is assumed to be low such that the liquid is confined to pendular rings, or "liquid islands", and no global flow of liquid occurs. Gas and vapor flow due to advection and diffusion can occur due to pressure, temperature, and/or concentration gradients.

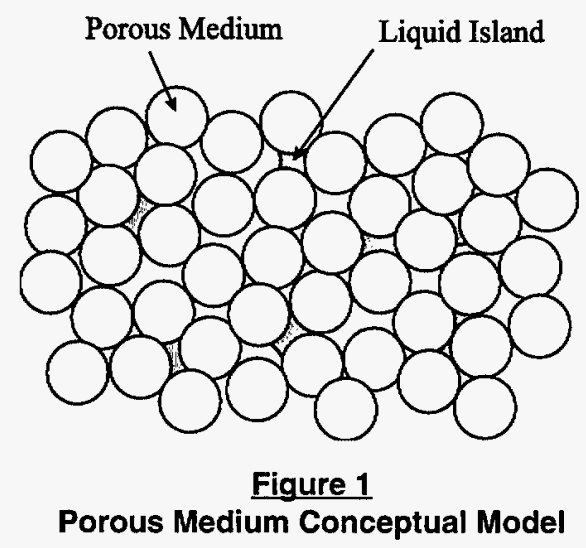

Simplified Representation. Figure 2 shows the simplified representation that was used in the present study based on the conceptual model shown in Figure 1. The particles are assumed to be arranged in rows, and the liquid islands are assumed to occur on a regular basis. Symmetry is invoked as indicated by the dashed-line box in the figure. A two-dimensional representation has been used for simplicity, and the solid particles are represented as cylinders.

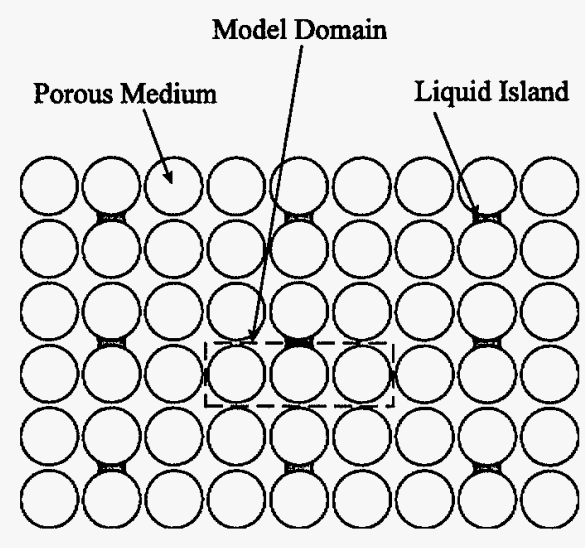

Figure 2

Porous Medium Simplified Representation

Numerical Method. For the present study, the TOUGH2 (Pruess, 1991) code has been employed. TOUGH 2 is a widely-used code for simulating fluid flow and heat transport in unsaturated fractured and porous media. Typical applications include environmental remediation, nuclear waste isolation, and geothermal processes (Pruess, 1995). TOUGH2 is an integrated finite difference code that solves mass, flux, and energy conservation equations in a porous media. The conservation equations 
used for the present study have been modified from the standard version of the code as summarized in the Appendix.

While TOUGH2 is technically a porous media code, it can also be envisioned as a code that simply solves the appropriate conservation equations. If the control volumes, or elements, have a porosity of 0 ., the element represents the solid phase. Similarly, if the elements have a porosity of 1.0 , the element represents a fluid region. Depending on the saturation of this fluid region, it can be all gas, all liquid, or a combination of the two. The advantage of TOUGH2 over conventional fluid flow codes is the inclusion of capillary pressure and vapor pressure lowering.

Other numerical approaches, including lattice-gas methods, could be applied to the present pore-scale problem. These methods may be more accurate than the present approach but at a cost of more model development time and effort. As discussed by Ho and Webb (1998), the existence of and the processes involved in enhanced vapor diffusion are still being evaluated. The first mechanistic calculation of enhanced vapor diffusion evaluating the processes involved was recently performed by Webb and Ho (1997). With the evaluation of the mechanisms involved in enhanced vapor diffusion, and experimental confirmation such as by Gu et al. (1998), development of a more accurate code may be appropriate. However, the pore-scale representation of the porous medium is itself a great simplification of the actual geometry. The present model is developed simply to investigate the various mechanisms at the pore scale which may lead to enhancement of vapor diffusion. As noted by Ewing and Gupta (1993), pore-scale modeling is a "useful concept rather than a physical reality". An approximate method, such as used in this paper, is an efficient approach for these initial investigations.

Model Geometry. Webb and Ho (1997) studied three different geometries in their investigation.

a. One-dimensional linear model,

b. Two-dimensional single pore, and

c. Two-dimensional pore network.

For the present study, their two-dimensional pore network model will be used because it considers multiple pores and a single liquid island. This model is the most realistic of the three developed by Webb and Ho (1997), in part because it considers the competition between vapor diffusion through open pores and through liquid islands.

\section{MODEL PARAMETERS} below.

The model parameters are summarized in Table 1 and are discussed

Pore-Scale Dimensions. The dimensions for the current pore-scale model are chosen to be consistent with the enhanced vapor diffusion data tabulated by Jury and Letey (1979). An average value of the capillary head (pressure) data is approximately $300 \mathrm{~cm}$ (range of $<10 \mathrm{~cm}$ to $5 \mathrm{x}$ $10^{4} \mathrm{~cm}$ with a median of $269 \mathrm{~cm}$ ). Using Young-Laplace's equation for the pressure difference across a curved surface, and an air-water surface tension, $\mathrm{F}$, at $20^{\circ} \mathrm{C}$ of 72.8 dynes $/ \mathrm{cm}$, a typical minimum pore radius is $5 \times 10^{-6} \mathrm{~m}$, or $5 \mu \mathrm{m}$.

The spheres are represented by an octagonal geometry; an octagon was selected rather than a square in order to approximate some of the variation of the pore cross-sectional area. A non-symmetrical octagon was employed such that the faces parallel to the $x$ and $y$ directions have a slightly different length than the diagonal faces. This shape allows for
Table 1

\section{Pore-Scale Model Parameters}

Dimensions

Pore Radius

Equivalent Permeability

Particle Radius

Model Porosity

Diffusion

Binary Diffusion Coefficient

Knudsen Diffusion Coefficient - Air

Knudsen Diffusion Coefficient - Vapor

$5 \mu \mathrm{m}$

$2 \times 10^{-12} \mathrm{~m}^{2}$

$50 \mu \mathrm{m}$

0.322

$2.42 \times 10^{-5} \mathrm{~m}^{2} / \mathrm{s}$

$1.54 \times 10^{-3} \mathrm{~m}^{2} / \mathrm{s}$

$1.96 \times 10^{-3} \mathrm{~m}^{2} / \mathrm{s}$

the use of a regular grid consisting of square elements except at the diagonal faces of the solid; square elements are desirable when using the 9-point scheme discussed below. On the diagonal faces, the square elements are divided into two equal triangles, one which is solid and one which is fluid. The particle radius in the numerical model has been chosen to be $50 \mu \mathrm{m}$, which supports the use of square elements and gives a reasonable model porosity value of 0.322 . The height and width of the octagon is $100 \mu \mathrm{m}$, consistent with the particle diameter.

The standard version of TOUGH 2 employs a 5-point stencil to connect the elements in the $\mathrm{x}$ - and $\mathrm{y}$-directions. This numerical scheme is not appropriate for flow along the diagonal surfaces in the model or for flow between the square and triangular elements. Therefore, a 9-point differencing scheme has been used which adds diagonal connections between elements. The main advantage of the 9-point scheme in the present model is that connections parallel to the diagonal surfaces of the solid particles allow for a more reasonable octagonal shape rather than a "stair-step" surface. Grid orientation effects can also be significant for the 5-point scheme, especially when a diagonal surface is present; these effects are greatly reduced when a 9-point scheme is employed as discussed by Webb and Ho (1997).

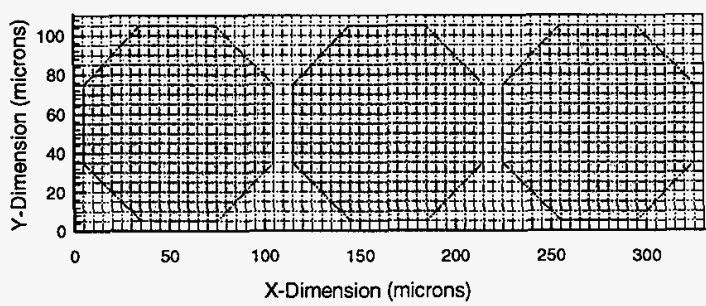

Figure 3

Two-Dimensional Pore Network Model

The nodalization of the two-dimensional pore network model is shown in Figure 3. The individual elements, or control volumes, are 5 $\mu \mathrm{m}$ on a side. The liquid island, if present, is located in the top pore above the center particle. For simplicity, the boundary element columns are not shown. The overall model is 68 elements long and 22 elements wide. The two end columns represent boundary conditions, so the effective dimensions are $330 \mu \mathrm{m}$ long and $110 \mu \mathrm{m}$ wide.

Permeability. The analogy between Darcy's law and laminar flow between parallel plates has been used (deMarsily, 1986), and the equivalent permeability for the minimum pore dimension of $5 \mu \mathrm{m}$ is $2 \mathrm{x}$ $10^{-12} \mathrm{~m}^{2}$. 
The analogy between Darcy's law and laminar flow between parallel plates is based on the distance between solid surfaces and results in a parabolic velocity profile based on a constant flow area. In the present model, the flow area varies dramatically in the direction of flow, so the suitability of a parabolic velocity profile is questionable. This problem has been at least partially addressed by Brown et al. (1995), who calculated velocity profiles between undulating surfaces of a hypothetical fracture. For the present particle geometry, the results from Brown et al. (1995) indicate that, under steady flow conditions, the fluid velocity profile will be nearly parabolic at the pore throat and "Gaussian" at the wider part of the channel. The fluid velocity is also dependent on the shape of the channel which is not captured in the analogy. Nevertheless, for simplicity, locally parabolic velocity profiles will be implicitly assumed in the present model by relying on the parallel plate analogy. Because the flow modeling is primarily concerned with diffusion, the error introduced through the use of this analogy should be small.

Accepting the parallel plate analogy for the present study, the effective permeability must vary normal to the flow direction in order to produce the desired parabolic velocity profile. For any given element, there may be different radii in the horizontal and vertical directions. Assuming parabolic profiles in both the horizontal and vertical directions, these radii result in different horizontal (h) and vertical (v) effective permeabilities.

Diffusion. The binary diffusion coefficient for the present study is 2.42 $x 10^{-5} \mathrm{~m}^{2} / \mathrm{s}$ at the analysis conditions of $10^{5} \mathrm{~Pa}$ and $20^{\circ} \mathrm{C}$ (Pruess, 1991). The Knudsen diffusion coefficient is calculated for perfectly diffuse molecule-wall collisions (coefficient of diffuse reflection $=1.0$ ). For the present minimum pore dimension of $5 \mu \mathrm{m}$, the Knudsen diffusion coefficient is $15.4 \mathrm{~cm}^{2} / \mathrm{s}$ or $1.54 \times 10^{-3} \mathrm{~m}^{2} / \mathrm{s}$ for air at $20^{\circ} \mathrm{C}$. For water vapor, the value for air can simply be scaled by the inverse of the square root of the ratio of molecular weights, resulting in a value of $1.96 \times 10^{-3}$ $\mathrm{m}^{2} / \mathrm{s}$. These values are a linear function of the local pore radius. No modifications of the diffusion coefficients are made to account for the presence of the porous medium because the present model is a pore-scale approach.

Similar to the effective permeability discussion, any given element may have different radii in the horizontal and vertical directions. However, as discussed in detail by Cunningham and Williams (1980), the fluid velocity from Knudsen diffusion is uniform and is independent of distance from the wall. The Knudsen diffusion coefficient is simply a function of the horizontal and vertical radii and, unlike the effective permeability, is not a function of the local coordinates.

Liquid Island. The model for the liquid island is one of the major pieces of the current pore-scale analysis. Capillary pressure across the gasliquid interface and vapor pressure lowering is included. Capillary pressure is a function of position, or length, of the liquid island as illustrated in Figure 4; the capillary pressure near the minimum pore dimension (A), which corresponds to a short liquid island, is much higher than for a much larger dimension (B), or a much longer liquid island. By geometry, the radius of curvature for a given contact angle can be calculated as a function of position, or equivalently, liquid island length. Assuming a contact angle of $0^{\circ}$, the capillary pressure as a function of position is shown in Figure 5, where the coordinate $(x)$ is zero at the minimum pore dimension. A maximum $x / R$ value of 0.75 , where $R$ is the particle radius, was used in the development of the capillary pressure function.

\section{Figure 4 \\ Schematic for the Liquid Island Capillary Pressure Function}

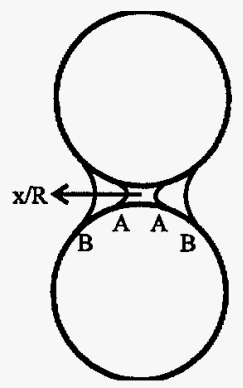

The capillary pressure due to the gas-liquid interface results in local vapor pressure lowering due to the curvature (Dullien, 1992). This effect can be quantified through Kelvin's equation which can be written as

$$
P_{v a i)}=f_{V P L} P_{s a t}
$$

where

$$
f_{V P L}=\exp \left(\frac{M_{w} P_{c}}{\rho_{w} R T}\right)
$$

where the capillary pressure is defined as $P_{\text {liquid }}$ minus $P_{g a s}$ which is negative. For the assumed uniform temperature of $20^{\circ} \mathrm{C}$, the saturated water vapor pressure is $2337 \mathrm{~Pa}$. For the maximum capillary pressure of about $-30 \mathrm{kPa}$ as shown in Figure 5, the vapor pressure lowering factor is 0.99978 , or a minimum vapor pressure of $2336.5 \mathrm{~Pa}$, which results in a maximum vapor pressure lowering of only about $0.5 \mathrm{~Pa}$.

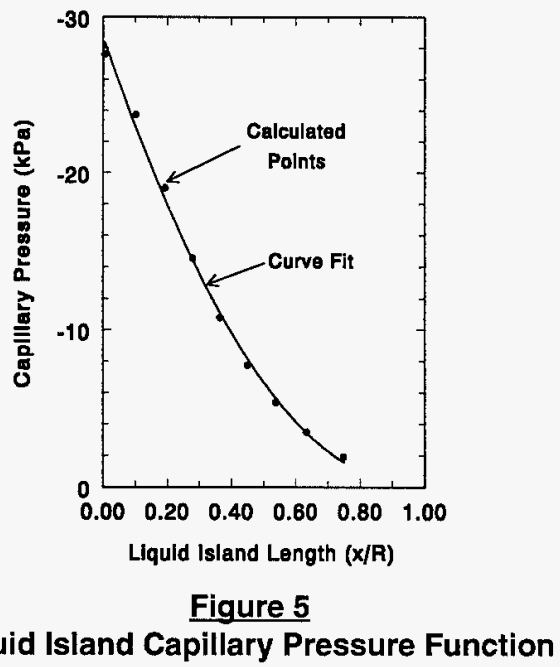

For the present simulations, only the longest liquid island is considered ( $\mathrm{x} / \mathrm{R}=0.75$ ). The resulting vapor pressure lowering is only about $0.033 \mathrm{~Pa}$, or $0.0014 \%$ of the saturated value. Even though the magnitude of vapor pressure lowering is small in magnitude and its effect is often ignored, it can have a large influence on enhanced vapor diffusion. For example, flow through liquid islands is driven by condensation and evaporation on both ends of the liquid island. Without vapor pressure lowering, steady-state enhanced vapor diffusion does not occur as discussed by Webb and Ho (1997). 
Boundary Conditions. The boundary conditions consist of left-hand and right-hand side boundary columns with uniform conditions in each column. A concentration gradient is imposed across the model for the present simulations; zero pressure and temperature gradients are assumed. Specification of the boundary conditions, including the magnitudes, and the influence of "assisting" or "opposing" boundary conditions is of considerable interest. These factors will be studied further as development of the pore-scale model evolves.

For steady-state vapor diffusion in which the flow rate into and out of the model are equal, the condensation and evaporation rates on the liquid island must be the same. For a zero concentration gradient, no condensation or evaporation should occur on the liquid island at equilibrium. This equilibrium condition is slightly dependent on the liquid island capillary pressure, and therefore the liquid island length, due to vapor pressure lowering effects. Due to vapor pressure lowering, the equilibrium vapor pressure is slightly lower than saturated vapor. A small concentration gradient, which is centered around this equilibrium condition, is then imposed to calculate steady-state vapor diffusion. The concentration gradient is sufficiently small such that the vapor pressure at the boundaries is always less than $\mathrm{P}_{\text {sat }}$ to avoid condensation and evaporation at locations other than the liquid island.

As the concentration gradient is increased, the higher vapor-pressure (higher relative humidity (RH)) boundary is limited by the saturation vapor pressure; this condition is the limit of steady-state vapor diffusion. In order to increase the gradient further, the lower vapor-pressure (lower $\mathrm{RH}$ ) boundary value is decreased resulting in transient drying boundary conditions. In this case, net evaporation occurs from the liquid island, and the mass flow rate into and out of the model are not equal. In the present calculations, the lower vapor-pressure boundary was reduced all the way to a zero vapor pressure (zero RH) condition. As will be seen, this combination of steady-state and transient drying calculations results in about a five order-of-magnitude variation in the vapor pressure (RH) gradient across the model.

In all cases, a false transient was performed to achieve quasi steadystate conditions such that mass flow rates were constant. This does not mean that the mass flow rates into and out of the model are necessarily the same; it simply means that the flow rates are slowly changing due to " change in the liquid island mass, and that the mass flow out of the model is the mass flow in plus the net evaporation rate from the liquid island.

Enhancement Factor. The enhancement factor for vapor diffusion is defined as the calculated vapor flux with a liquid island divided by the vapor flux for all-gas conditions. The vapor flux for all-gas conditions is easily calculated by simply removing the liquid island. Similarly, for a liquid island under steady-state boundary conditions, the vapor fluxes into and out of the model are the same, and the enhancement factor is simply the vapor flux divided by the all-gas flux for the same boundary conditions.

However, for transient drying conditions, the situation is more complicated. The vapor flux is spatially variable, i.e., the vapor flux into the model is not equal to the vapor flux out of the model due to net liquid island evaporation. For the purposes of defining an average enhancement factor, the average of the mass flux into and out of the model is used. As will be seen, this definition has many advantages.

\section{RESULTS}

The basic model used in the present study was shown earlier in Figure 3. A liquid island is located in the top center throat of the model. As mentioned above, with vapor pressure lowering, the equilibrium vapor pressure at either end of the liquid island is only reduced by about 0.033 $\mathrm{Pa}$, resulting in an equilibrium relative humidity of 99.9986 percent.

As discussed earlier, both steady-state and transient conditions are considered. Steady-state occurs when the boundary conditions are centered around the liquid island condition such that condensation and evaporation rates are equal. The range of boundary relative humidities is dramatically limited for this situation such that the minimum relative humidity $(\mathrm{RH})$ value of the lower vapor pressure boundary is about 99.9972 percent (with a higher vapor pressure boundary condition $\mathrm{RH}$ of 100 percent). For transient conditions, in which the condensation and evaporation rates are not equal, the lower RH boundary can decrease all the way to 0 . This boundary condition imposes a severe vapor pressure gradient which results in strong evaporation from both ends of the liquid island.

With Vapor Pressure Lowering. Steady-state vapor diffusion results for the vapor velocity vectors are shown in Figure 6 for all-gas conditions and for two different RH differences. The liquid island is located in the top pore of the center particle. The velocity vectors are normalized with respect to the RH difference to facilitate comparisons. The plots are practically identical for the two RH differences shown and indicate that vapor "diffuses" through the liquid island, similar to Webb and Ho (1997). More vapor diffuses through the liquid island than through the open pore on the opposite side of the center particle even though the pore geometries are the same. This behavior is caused by the liquid island which reduces the effective diffusion path for the vapor and actually "pulls" vapor towards it. As the RH difference is increased further, transient vapor diffusion occurs, and there is net evaporation from the liquid island as shown in the vector plots in Figure 7. These velocity vectors are different than for steady-state due to the significant net evaporation from the liquid island, especially at the downstream end. Eventually, there is evaporation from both ends of the liquid island due to the strong vapor pressure gradient.

The results from the various simulations can be summarized by plotting the enhancement of the vapor mass flux across each boundary as well as the average value as depicted in Figure 8 . The enhancement value is defined as the vapor mass flux across a boundary divided by the vapor mass flux across the boundary for all-gas conditions with the same boundary conditions. For steady-state boundary conditions, the vapor mass flux across the lower and higher RH boundaries is the same by definition, and the value of the enhancement factor is constant at about 1.3. (Note that if more liquid islands were present, the enhancement factor would be much larger. For example, Webb and Ho (1997) calculated enhancement factors greater than 10.) With transient boundary conditions, the enhancement factors for each boundary change, but somewhat surprisingly, the average value remains constant and equal to the steady-state boundary results. Therefore, based on these simulations, enhancement factors based on the average vapor mass flux are the same for steady-state and transient conditions. These results imply that the enhancement factor data obtained by $\mathrm{Gu}$ et al. (1998) were not affected 


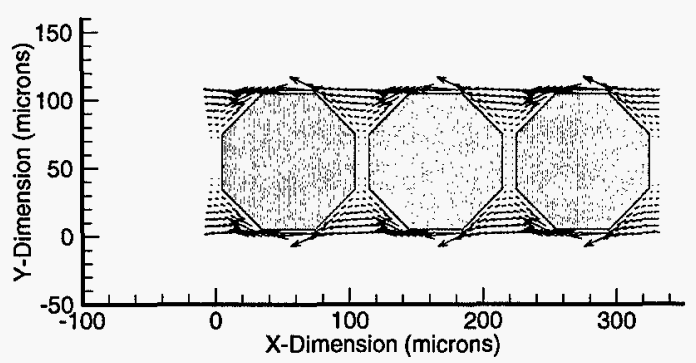

(a) All-Gas Conditions - $\Delta \mathrm{RH}=0.001 \%$

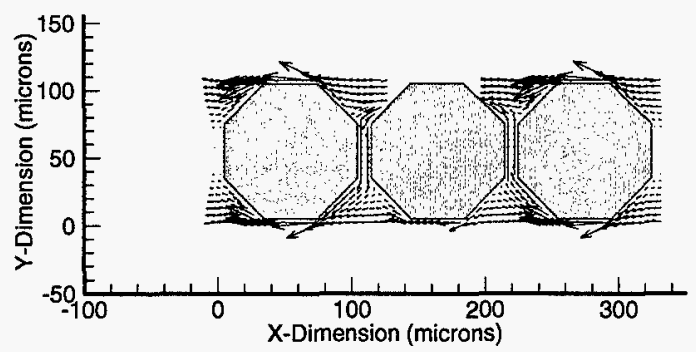

(b) With Liquid Island - $\Delta \mathrm{RH}=0.001 \%$

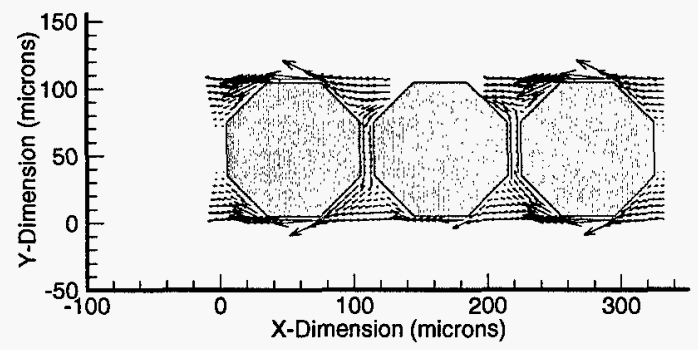

(c) With Liquid lsland - $\Delta \mathrm{RH}=0.002 \%$

\section{Figure 6}

Velocity Vectors with Vapor Pressure Lowering for SteadyState Boundary Conditions

by the transient nature of the experiments as long as the average vapor mass flux is used.

The influence of the boundary conditions on the net evaporation rate from the liquid island is important in determining the liquid evolution for transient conditions. If net evaporation occurs, the liquid island will disappear with time. Figure 9 shows the net liquid island evaporation rate (mass flux to lower RH boundary minus mass flux from higher RH boundary) normalized to the all-gas diffusion rate. For steady-state conditions, no net evaporation occurs, so any liquid island would persist. The vapor mass flux from the higher RH boundary and the mass flux to the lower RH boundary are equal. As indicated by the velocity vector plots, a significant amount of vapor "diffuses" through the liquid island. As the RH difference is increased into the transient regime, net evaporation increases considerably such that most of the flow is due to evaporation of the liquid island.

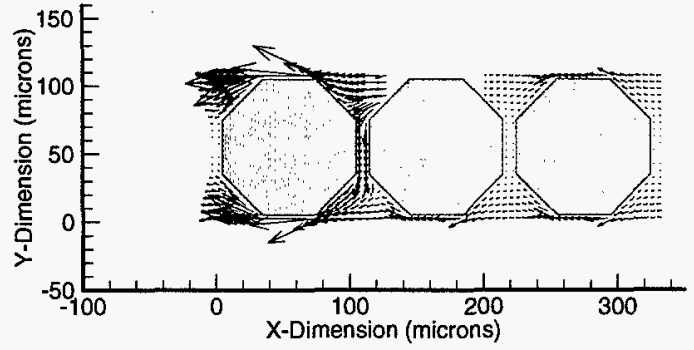

(a) With Liquid Island $\cdot \Delta \mathrm{RH}=0.006 \%$

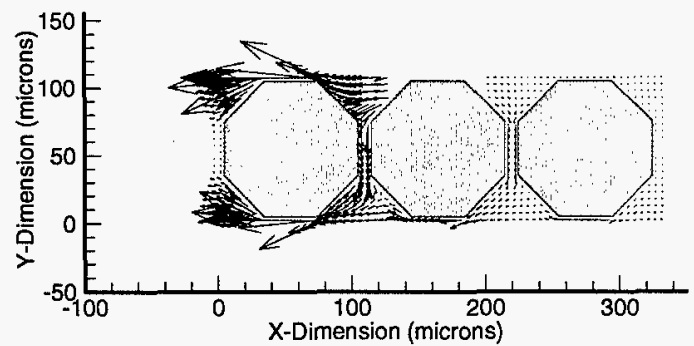

(b) With Liquid Island - $\Delta \mathrm{RH}=0.1 \%$

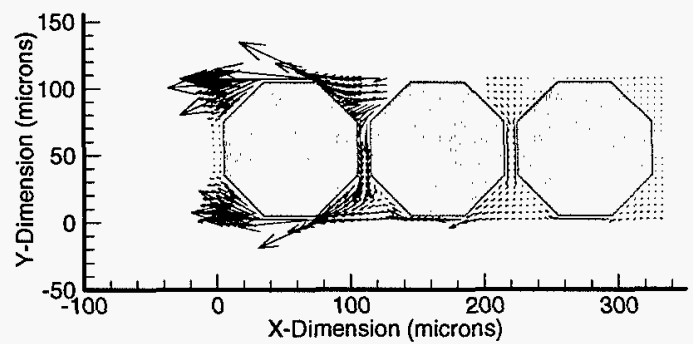

(c) With Liquid Island - $\Delta \mathrm{RH}=100 \%$

\section{Figure 7 \\ Velocity Vectors with Vapor Pressure Lowering for Transient Boundary Conditions}

Without Vapor Pressure Lowering. Vapor pressure lowering is often ignored in pore-scale models. The effect of neglecting vapor pressure lowering on the above simulation results is discussed below.

Vapor diffusion results for the vapor velocity vectors are shown in Figure 10 for the same conditions as Figure 6. As mentioned earlier, steady-state conditions do not exist without vapor pressure lowering. For the lower RH difference, evaporation occurs from both ends of the liquid island. As the RH difference increases, evaporation shifts toward the lower RH end of the liquid island. (With vapor pressure lowering, net evaporation did not occur for these RH differences.) For transient boundary conditions, the velocity vectors given in Figure 11 indicate that evaporation still occurs predominantly from the lower RH end of the liquid island. The results for the larger RH differences are practically identical to those with vapor pressure lowering. In summary, for steadystate and the lower RH difference transient boundary conditions, the results are significantly different with and without vapor pressure lowering. For transient conditions at the higher RH differences, the results with and without vapor pressure lowering converge. 


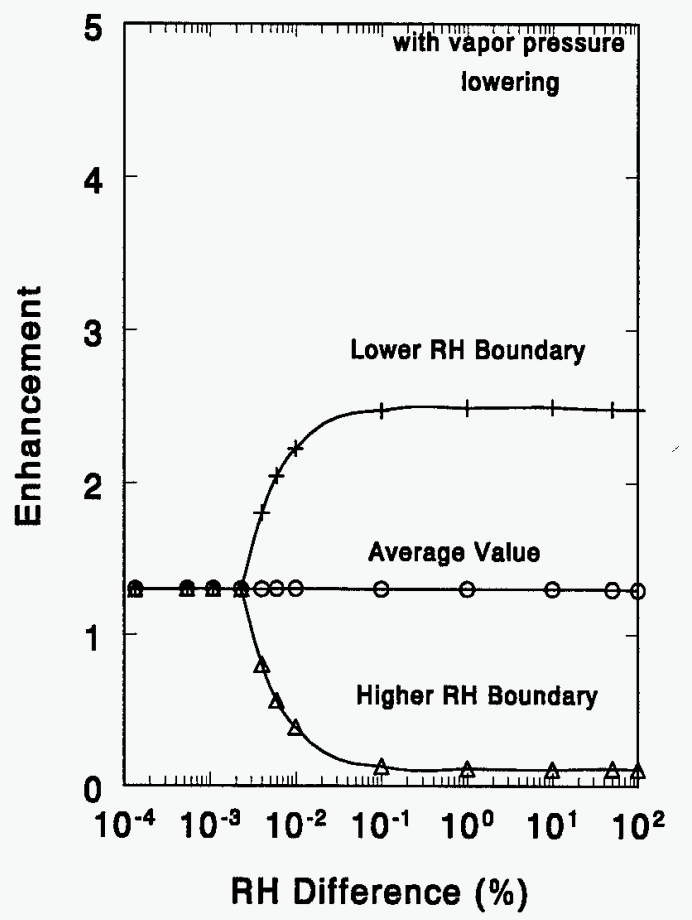

\section{Figure 8}

\section{Enhancement Factors with Vapor Pressure Lowering}

The enhancement factors from the various simulations without vapor pressure lowering are shown in Figure 12; the factors with vapor pressure lowering are also included for comparison purposes. While the average value of the enhancement factor is constant and is the same with or without vapor pressure lowering, the behavior of the boundary enhancement factors differs dramatically. For steady-state conditions without vapor pressure lowering, the enhancement factor is negative for small RH differences indicating vapor flow toward the higher RH boundary. The RH of the liquid island is 1.0 because vapor pressure lowering is neglected. For transient conditions, the enhancement factors are independent of $\mathrm{RH}$ difference. At the higher $\mathrm{RH}$ differences, the enhancement factors with and without vapor pressure lowering converge.

The influence of the boundary conditions on the net evaporation rate from the liquid island is important as shown in Figure 13; results with and without vapor pressure lowering are included. For steady-state conditions without vapor pressure lowering, the evaporation rate is large and decreases with increasing RH difference; in contrast, the net evaporation rate with vapor pressure lowering is zero. For transient conditions, the evaporation rate without vapor pressure lowering is constant. The results with and without vapor pressure lowering converge as the RH difference increases.

\section{DISCUSSION}

Enhanced vapor diffusion occurs under steady-state and transient conditions. While the details of the pore-scale behavior are a function of steady-state or transient conditions and the magnitude of the RH difference, the average enhancement factor based on the average mass flux is constant. These results have important implications on experimental and modeling efforts. As discussed in Gu et al. (1998),

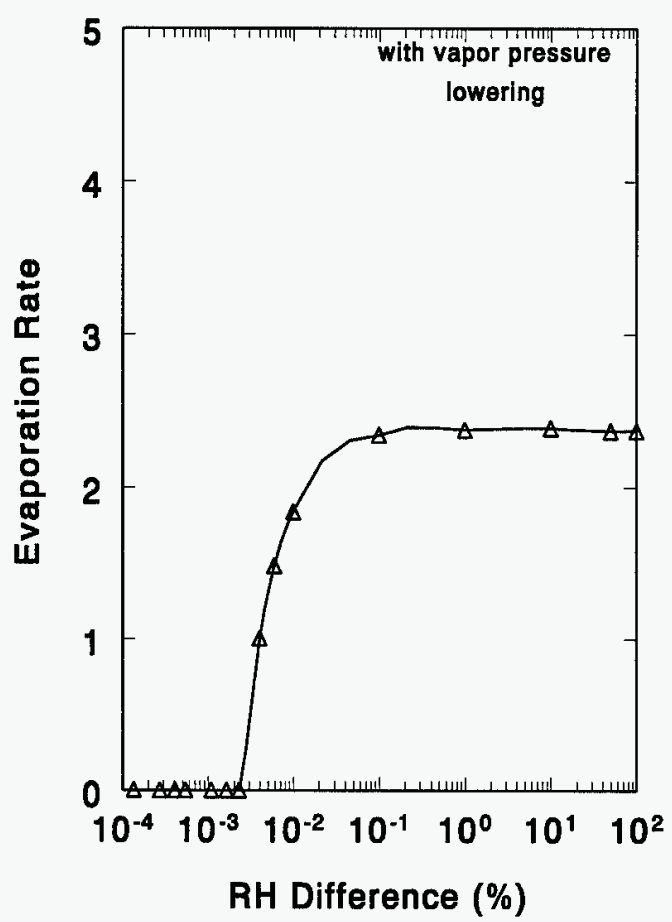

\section{Figure 9 \\ Evaporation Rate with Vapor Pressure Lowering}

steady-state vapor diffusion experiments are difficult to perform, and only transient data were obtained. Based on the present study, their enhancement factors using the average vapor mass flux do not need to be corrected for transient effects and have the same numerical value as for steady-state conditions. These results also simplify the modeling effort because evaporation and vapor diffusion mass flow rates can be combined using a single enhancement factor.

In many previous analyses of vapor diffusion, vapor pressure lowering has been ignored. While the magnitude of vapor pressure lowering is numerically small compared to the total vapor pressure, the effect on vapor diffusion on the pore scale can be significant as shown in this study. Vapor pressure lowering can dramatically influence the liquid island evolution as indicated by the net liquid island evaporation rate, especially for small RH differences.

The magnitude of the vapor diffusion enhancement factor is a function of many different parameters, and the value given in this paper should not be considered to be representative of all conditions. For example, Webb and Ho (1997) calculated enhancement factors up to and greater than 10 . The magnitude also changes dramatically with the location of the liquid island. For example, in the present model, if the liquid island were moved to the pore next to the lower RH boundary, the average enhancement factor would jump from 1.30 to 7.65 .

It should be kept in mind that the present results are with a concentration gradient only; there are no temperature or pressure gradients. In addition, the RH gradient is imposed over three particle diameters, or $330 \mu \mathrm{m}$. It also must be noted that these conclusions are based on modeling results only, and that pore-scale modeling is only "a useful concept rather than a physical reality". Nevertheless, these results indicate that vapor pressure lowering should be considered in future porescale modeling efforts. 


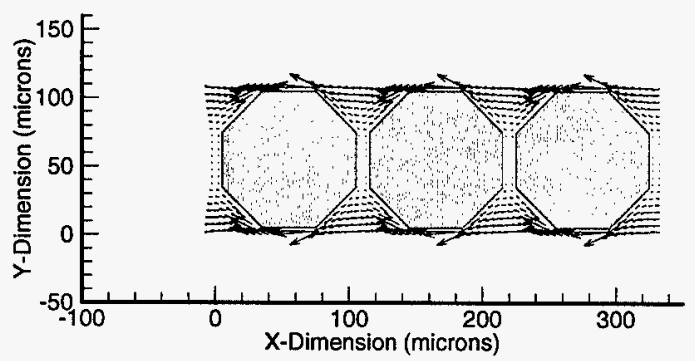

(a) All-Gas Conditions - $\triangle \mathrm{RH}=0.001 \%$

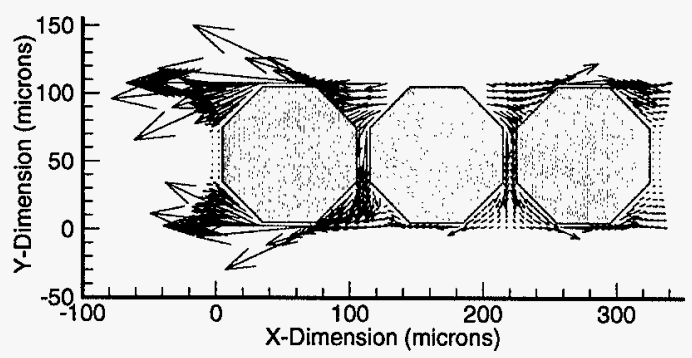

(b) With Liquid island - $\triangle \mathrm{RH}=0.001 \%$

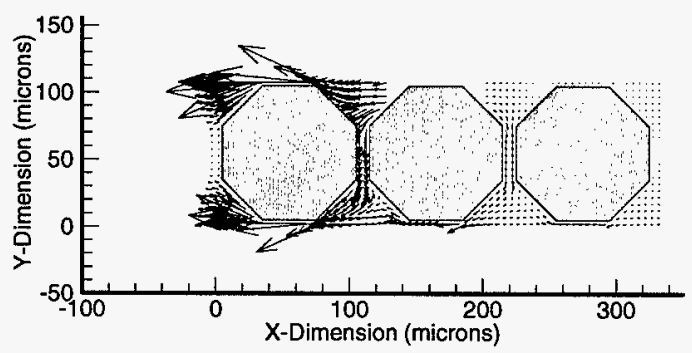

(c) With Liquid Island - $\Delta \mathrm{RH}=0.002 \%$

Figure 10

Velocity Vectors without Vapor Pressure Lowering for Steady-State Boundary Conditions

\section{CONCLUSIONS}

The conclusions from the present pore-scale modeling study of vapor diffusion under partially-saturated conditions under a concentration gradient are:

1.The details of vapor diffusion on the pore scale in the presence of its own liquid are strongly affected by the boundary conditions of the model. The flow patterns and enhancement factors across the boundaries vary with the magnitude of the RH difference across the model.

2. The enhancement factor for vapor diffusion based on the average mass flux is constant with respect to boundary conditions. Therefore, data such as Gu et al. (1998) do not need to be corrected for transient effects if the enhancement factors are based on the average value.

3. Vapor pressure lowering should be considered in pore-scale analysis of vapor diffusion in the presence of its own liquid. While the magnitude of vapor pressure lowering may be small compared to the total vapor pressure, it can have significant impact on local vapor condensation, evaporation, and the evolution of liquid islands within the pores.

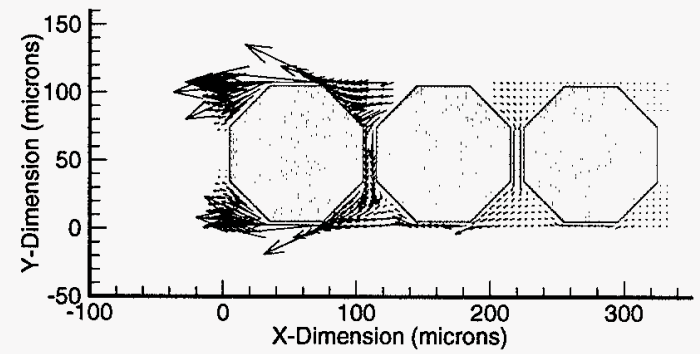

(a) With Liquid Island - $\Delta \mathrm{RH}=0.006 \%$

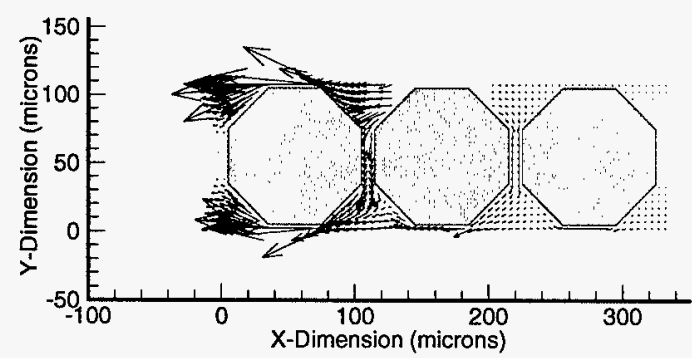

(b) With Liquid Island - $\Delta \mathrm{RH}=0.1 \%$

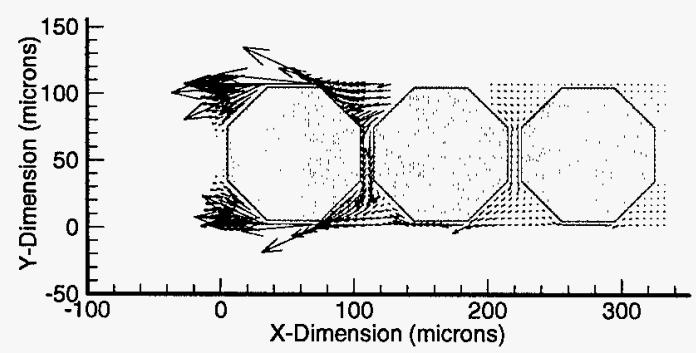

(c) With Liquid Island - $\Delta \mathrm{RH}=100 \%$

Figure 11

Velocity Vectors without Vapor Pressure Lowering for Transient Boundary Conditions

\section{ACKNOWLEDGMENTS}

This work was supported by the United States Department of Energy under Contract DE-AC04-94AL85000 as part of a Sandia Laboratory Directed Research and Development Project on Enhanced Vapor-Phase Diffusion in Porous Media. Sandia is a multiprogram laboratory operated by Sandia Corporation, a Lockheed Martin Company, for the United States Department of Energy.

\section{REFERENCES}

Bird, R.B., Stewart, W.E., and Lightfoot, E.N., 1960, Transport Phenomena, John Wiley \& Sons, New York.

Brown, S.R., Stockman, H.W. and Reeves, S.J.,1995, "Applicability of the Reynolds equation for modeling fluid flow between rough surfaces," Geo. Res. Let., 22:2537-2540.

Cary, J.W., 1979, "Soil heat transducers and water vapor flow," Soil Sci. Soc. Am. J., 43:835-839.

Cass, A., Campbell, G.S., Jones, T.L., 1984, "Enhancement of Thermal Water Vapor Diffusion in Soil," Soil Sci, Soc. Am. J., 48:25-32. 


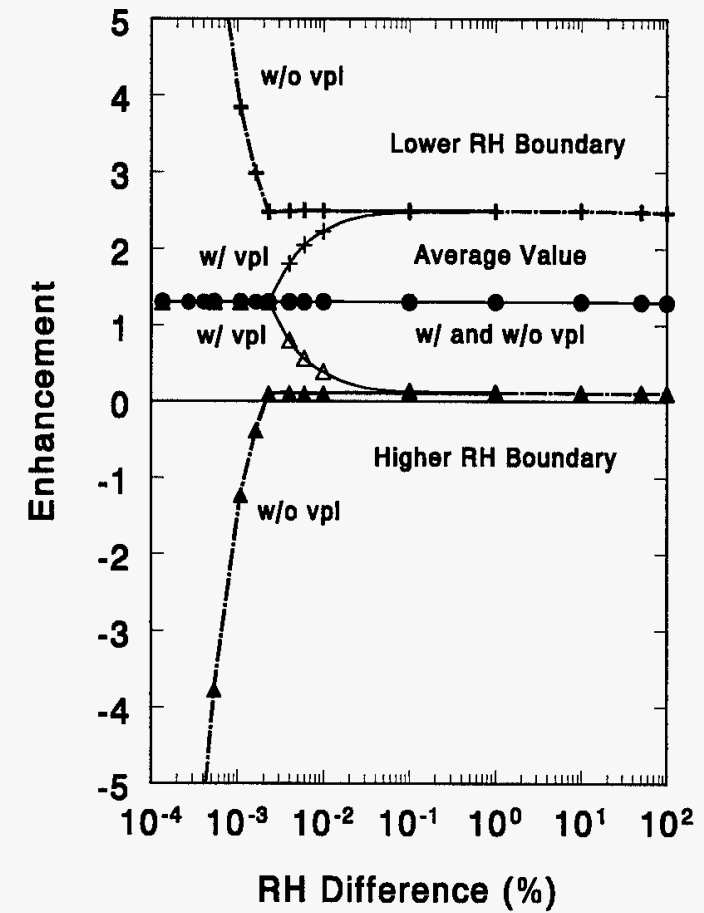

Figure 12

Enhancement Factors with and without Vapor Pressure Lowering

Cunningham, R.E., and Williams, R.J.J., 1980, Diffusion in Gases and Porous Media, Plenum Press, New York.

de Marsily, G., 1986, Quantitative Hydrogeology Groundwater Hydrology for Engineers, Academic Press, Inc., San Diego.

Dullien, F.A.L., 1992. Porous Media Fluid Transport and Pore Structure, Second Edition, Academic Press, Inc., San Diego.

Ewing, R.P., and Gupta, S.C. 1993, "Modeling percolation properties of random media using a domain network," Water Resourc. Res., 29:3169-3178.

Gu, L., Ho, C.K., Plumb, O.A., and Webb, S.W., 1998, "Diffusion with Condensation and Evaporation in Porous Media," 1998 AIAAASME Thermophysics and Heat Transfer Conference.

Ho, C.K., and Udell, K.S. 1992, "An experimental investigation of air venting of volatile liquid hydrocarbon mixtures from homogeneous and heterogeneous porous media," J. Contam. Hydrol., 11:291-316.

Ho, C.K., and Webb, S.W. 1998, "A Review of Porous Media Enhanced Vapor-Phase Diffusion Mechanisms, Models, and Data - Does Enhanced Vapor-Phase Diffusion Exist?" J. Porous Media, 1:71-92.

Jury, W.A., and Letey, Jr., J., 1979, "Water Vapor Movement in Soil: Reconciliation of Theory and Experiment," Soil Sci. Soc. Am. J., 43:823-827.

Mason, E.A., and Malinauskas, A.P., 1983, Gas Transport in Porous Media: The Dusty-Gas Model, Chem Eng. Monograph 17, Elsevier, New York.

Nowicki, S.C., Davis, H.T., and Scriven, L.E., 1992, “Microscopic Determination of Transport Parameters in Drying Porous Media," Drying Technology, 10:925-946.

Philip, J.R., and deVries, D.A., 1957, "Moisture Movement in Porous Materials under Temperature Gradients," Trans., Am. Geophys. Union, 38:222-232, 594.

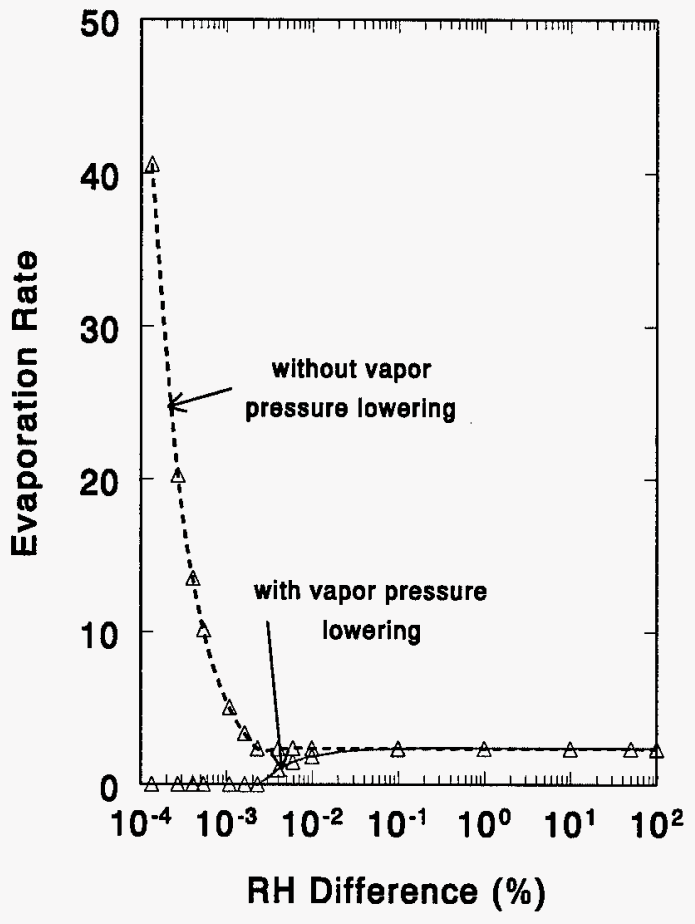

\section{Figure 13 \\ Evaporation Rate with and without Vapor Pressure Lowering}

Plumb, O.A., and Prat, M., 1992, "Microscopic Models for the Study of Drying of Capillary Porous Media," Drying '92, pp. 397-406.

Prat, M., 1993, "Percolation Model of Drying Under Isothermal Conditions in Porous Media,", Int. J. Multiphase Flow, 19:691-704.

Pruess, K., 1991, TOUGH2 - A General-Purpose Numerical Simulator for Multiphase Fluid and Heat Flow, LBL-29400, Lawrence Berkeley Laboratory.

Pruess, K., 1995, Proceedings of the TOUGH Workshop '95, LBL37200, Lawrence Berkeley Laboratory.

Pruess, K., and Narasimhan, T.N., 1985, "A Practical Method for Modeling Fluid and Heat Flow in Fractured Porous Media," SPE Journal, 25:14-26.

Webb, S.W., 1998, "Gas-Phase Diffusion in Porous Media Evaluation of an Advective-Dispersive Formulation and the Dusty-Gas Model," J. Porous Media (in press).

Webb, S.W., and C.K. Ho, 1997, "Pore-Scale Modeling of Enhanced Vapor Diffusion in Porous Media," Sixth Symposium on Multiphase Transport in Porous Media, 1997 ASME International Mechanical Engineering Congress and Exhibition, November 16-21, 1997.

Webb, S.W., and Phelan, J.M., 1997, "Effect of Soil Layering on NAPL Removal Behavior in Soil-Heated Vapor Extraction," J. Contam. Hydrol., 27:285-308.

Whitaker, S., 1977, "Simultaneous heat, mass and momentum transfer in porous media. A theory of drying." Advances in Heat Transfer, Vol. 13, Academic Press, New York. 


\section{Appendix TOUGH2 Conservation Equations}

The formulation for an arbitrarily-shaped domain, $\mathrm{V}$, is as follows for the accumulation, flux, and source terms (Pruess and Narasimhan, 1985):

$$
\frac{d}{d t} \int_{V} M_{\kappa} d V=\int_{\Gamma} F_{\kappa} \cdot n d \Gamma+\int_{V} q_{\kappa} d V
$$

where $\kappa$ is water, air, or heat. The fluid mass accumulation term is

$$
M_{\kappa}=\phi \sum_{\beta=1}^{2} S_{\beta} \rho_{\beta} \omega_{\beta, \kappa}
$$

where $\beta$ is the liquid or the gas phase. The heat accumulation term is

$$
M_{3}=(1-\phi) \rho_{R} C_{R} T+\phi \sum_{\beta=1}^{2} S_{\beta} \rho_{\beta} u_{\beta}
$$

$$
D^{*}=D_{\mathrm{\kappa \kappa}}+x_{\kappa} D_{\kappa^{*}}^{K}+x_{\kappa^{*}} D_{\kappa}^{K}
$$

The above equations for the Dusty Gas Model are written in terms of mole fraction rather than mass fraction because mole fraction is a more natural variable for diffusion.

In equation 7 , the flux of component $\kappa$ consists of a diffusive flux (first and second terms) and an advective flux (third term). The diffusive flux has ordinary diffusion (mole fraction gradient) and Knudsen diffusion (pressure gradient) components.

The flux term for the liquid phase is simply

$$
F_{l}=-\frac{k}{\mu_{l}} \rho_{l} \omega_{l, \kappa}\left(\nabla P_{l}-\rho_{l} g\right)
$$

where the assumption of separation of phases has been used (relative permeability $=1$.).

The heat flux term is simply

$$
F_{3}=-K \nabla T+\sum_{\substack{\beta=l, g \\ \mathrm{\kappa}=1.2}} h_{\beta, \mathrm{\kappa}} F_{\beta, \mathrm{\kappa}}
$$

The flux term for the gas phase is based on the Dusty Gas Model (DGM) (Mason and Malinauskas, 1983), which considers advection, Fickian diffusion, and Knudsen diffusion, including the coupling between the various mechanisms. The original version of TOUGH2 uses an advective-dispersive formulation in which the fluxes from the various mechanisms is simply added together; the coupling among the mechanisms is not considered. The difference between these two formulations is discussed in detail by Webb (1998) including quantification of the differences in the various mechanisms. For the DGM, the gas mass flux of component $\kappa$ in the gas phase for a binary system can be written as

$$
\begin{aligned}
F_{\kappa} & =-J_{\kappa} M_{\kappa}=-M_{\kappa} \frac{D_{\kappa}^{K} D_{\kappa \kappa}{ }^{*} \frac{P_{g}}{R T} \nabla x_{\kappa}}{D^{*}} \\
& -M_{\kappa} \frac{D_{\kappa}^{K}\left(D_{\kappa \kappa^{*}}+D_{\kappa}{ }^{K}\right) x_{\kappa} \frac{\left(\nabla P_{g}-\rho_{g} g_{c}\right)}{R T}}{D^{*}} \\
& -M_{\kappa} x_{\kappa} \frac{k P_{g}}{\mu_{g}} \frac{\left(\nabla P_{g}-\rho_{g} g_{c}\right)}{R T}
\end{aligned}
$$

Capillary pressure and vapor pressure lowering effects can be included in TOUGH2. By setting the control volume porosity equal to 0.0 or 1.0 , the control volumes can be used to represent solid or fluid regions. 


\section{M98005451}

|||||||||||||||||||||||||||||||||||||||||||||

$$
\text { Report Number (14) } \frac{\text { SAND--98-1061C }}{\text { CONF-980610 }}
$$

Publ. Date (11) 199805
Sponsor Code (18) $D 0 E / M A, X F$
UC Category (19) UC-900, DOE/ER 